07

\title{
Структурно-энергетические закономерности накопления повреждений при деформировании гетерогенного материала
}

\author{
() Е.Е. Дамаскинская ${ }^{1}$, И.А. Пантелеев², Д.В. Корост ${ }^{3}$, К.А. Дамаскинский \\ ${ }^{1}$ Физико-технический институт им. А.Ф. Иофрфе РАН, \\ Санкт-Петербург, Россия \\ ${ }^{2}$ Институт механики сплошных сред УрО РАН, \\ Пермь, Россия \\ ${ }^{3}$ Московский государственный университет им. М.В. Ломоносова, \\ Москва, Россия \\ ${ }^{4}$ Санкт-Петербургский политехнический университет Петра Великого, \\ Санкт-Петербург, Россия \\ E-mail: Kat.Dama@mail.ioffe.ru
}

Поступила в Редакцию 7 сентября 2020 г.

В окончательной редакции 7 сентября 2020 г.

Принята к публикации 11 сентября 2020 г.

\begin{abstract}
Проведено экспериментальное исследование накопления дефектов при одноосном сжатии природного гетерогенного материала. Контроль процесса дефектообразования осуществлялся с помощью двух методов неразрушающего контроля: акустической эмиссии (AЕ) и рентгеновской компьютерной микротомографии (CT). Совместное использование этих методов позволило установить соответствие между энергетическими характеристиками $\mathrm{AE}$, сопровождающей образование дефектов, и объемом этих дефектов. Установлено, что зависимость объема дефектов и суммарной энергии сигналов АЕ имеет линейный вид, что соответствует ранее полученным феноменологическим зависимостям для очагов тектонических землетрясений. На основе полученной линейной зависимости проведена оценка среднего размера дефекта. Показано, что вне зависимости от предположений о форме дефекта, его средний линейный размер не превышает $100 \mu \mathrm{m}$.
\end{abstract}

Ключевые слова: акустическая эмиссия, рентгеновская компьютерная томография, объем дефектов, b-value анализ.

DOI: 10.21883/FTT.2021.01.50406.189

\section{1. Введение}

Формирование и развитие разномасштабных трещин в механически нагруженном материале сопровождается выделением тепловой, акустической и электромагнитной энергии, связанным с разрывом межатомных связей, образованием новых поверхностей и сопутствующими процессами неупругого деформирования [1-10]. При этом пропорция диссипированной и запасенной в деформируемом материале энергии на различных этапах нагружения определяется как внутренними (состав, кристаллическая структура, дефектность), так и внешними (интенсивность и конфигурация приложенных нагрузок, температура) факторами [11-14]. В случае квазистатического деформирования хрупких гетерогенных материалов преобладающим механизмом диссипации энергии является генерация упругих волн (акустическая эмиссия $(\mathrm{AE}))$, сопровождающая появление и развитие субмикро-, микро- и макротрещин. Анализ параметров потока сигналов акустической эмиссии является одним из широко используемых методов неразрушающего контроля, позволяющим контролировать весь объем деформируемого материала и выявлять наиболее опасные дефекты, развитие которых может привести к потери целостности объекта.
Одной из наиболее важных задач акустической эмиссии как физического явления является вопрос взаимосвязи геометрических размеров источника акустической эмиссии и энергии, выделившейся в виде упругих волн. Для различных пространственных размеров источников упругих волн (от микротрещин до разломов в Земной коре) накоплен целый ряд эмпирических соотношений между их геометрическими размерами (длиной, площадью, объемом) и параметрами, отражающими активность процесса генерации упругих волн (интенсивность $\mathrm{AE}$, энергия, сброшенные напряжения, и др.). Необходимо отметить, что применительно к лабораторным образцам, как правило, эти соотношения получены либо для одиночной трещины и связывают скорость ее роста с интенсивностью АЕ [15-17], либо определены для ансамбля поверхностных трещин в окрестности концентратора напряжений и связывают их количество (или плотность) с интегральными и дифференциальными параметрами акустической эмиссии (суммарный счет, скорость генерации АЕ) [18-21]. Для более общего случая накопления трещиноподобных дефектов в объеме деформируемого материала в отсутствии макроскопических концентраторов напряжений вопрос связи размеров дефектов (или их приращения) и энергии генерируемой при этом акустической эмиссии до сих пор остается от- 
Минеральный состав песчаника Berea

\begin{tabular}{c|c|c|c|c|c}
\hline Минерал & $\begin{array}{c}\text { Кварц } \\
\text { (quartz) }\end{array}$ & $\begin{array}{c}\text { Плагиоклаз } \\
\text { (plagioglase) }\end{array}$ & $\begin{array}{c}\text { Полевой шпат } \\
\text { (k-feldspar) }\end{array}$ & $\begin{array}{c}\text { Силикат алюминия } \\
\text { (kaolinite) }\end{array}$ & $\begin{array}{c}\text { Кальцит } \\
\text { СаСО } \\
\text { (calcite) }\end{array}$ \\
\hline & $85-90 \%$ & $1-2 \%$ & $3-6 \%$ & $2-6 \%$ & $6-8 \%$ \\
\hline Плотность & $2600-2650 \mathrm{~kg} / \mathrm{m}^{3}$ & $2620-2760 \mathrm{~kg} / \mathrm{m}^{3}$ & $2560 \mathrm{~kg} / \mathrm{m}^{3}$ & $2600 \mathrm{~kg} / \mathrm{m}^{3}$ & $2710 \mathrm{~kg} / \mathrm{m}^{33}$
\end{tabular}

крытым. Практическая значимость выявления подобной взаимосвязи заключается в возможности оценки характерных размеров дефектов, образующихся в процессе эксплуатации объекта контроля, по энергии регистрируемой акустической эмиссии.

Целью настоящей работы является установление корреляции между параметрами акустической эмиссии, регистрируемой в процессе квазистатического сжатия природного гетерогенного материала, и его дефектной структурой. Отличительной особенностью данной работы является совместное использование двух методов неразрушающего контроля: акустической эмиссии и рентгеновской компьютерной томографии (СТ), позволяющей с высоким разрешением визуализировать внутреннюю структуру материала.

\section{2. Методика эксперимента}

В качестве материала для исследований была выбрана хорошо изученная хрупкая осадочная порода - песчаник Berea [22]. Минеральный состав песчаника представлен в таблице. Песчаник Berea преимущественно сложен зернами кварца с характерным размером $20 \mu \mathrm{m}$. Образцы имели цилиндрическую форму с диаметром $10 \mathrm{~mm}$ и отношением размеров $h / d=2$. Перед экспериментами торцы образцов дополнительно шлифовались для обеспечения их плоскопараллельности. Боковая поверхность образцов перед экспериментами фиксировалась слоем клейкой пленки для предотвращения их рассыпания в процессе деформирования.

Программа нагружения образцов песчаника Berea состояла из двух этапов. На первом этапе образец подвергался квазистатическому одноосному сжатию со скоростью нагружения (смещения нагружающих плит) $5 \mu \mathrm{m} / \mathrm{min}$. Сжатие осуществлялось до усилия, равного 0.9 от $F_{\max }\left(F_{\max }-\right.$ разрушающая нагрузка, определенная в предварительных экспериментах). Затем образец выдерживался при постоянной деформации до тех пор, пока активность АЕ не спадала до нуля.

Регистрация акустической эмиссии в процессе нагружения песчаника осуществлялась с помощью системы Amsy-5 Vallen (Германия). Два пьезопреобразователя AE105A (рабочий частотный диапазон 450-1150 kHz) крепились в специальных полых цилиндрических плитах, с помощью которых непосредственно осуществлялось нагружение образца (рис. 1). Подобное размещение

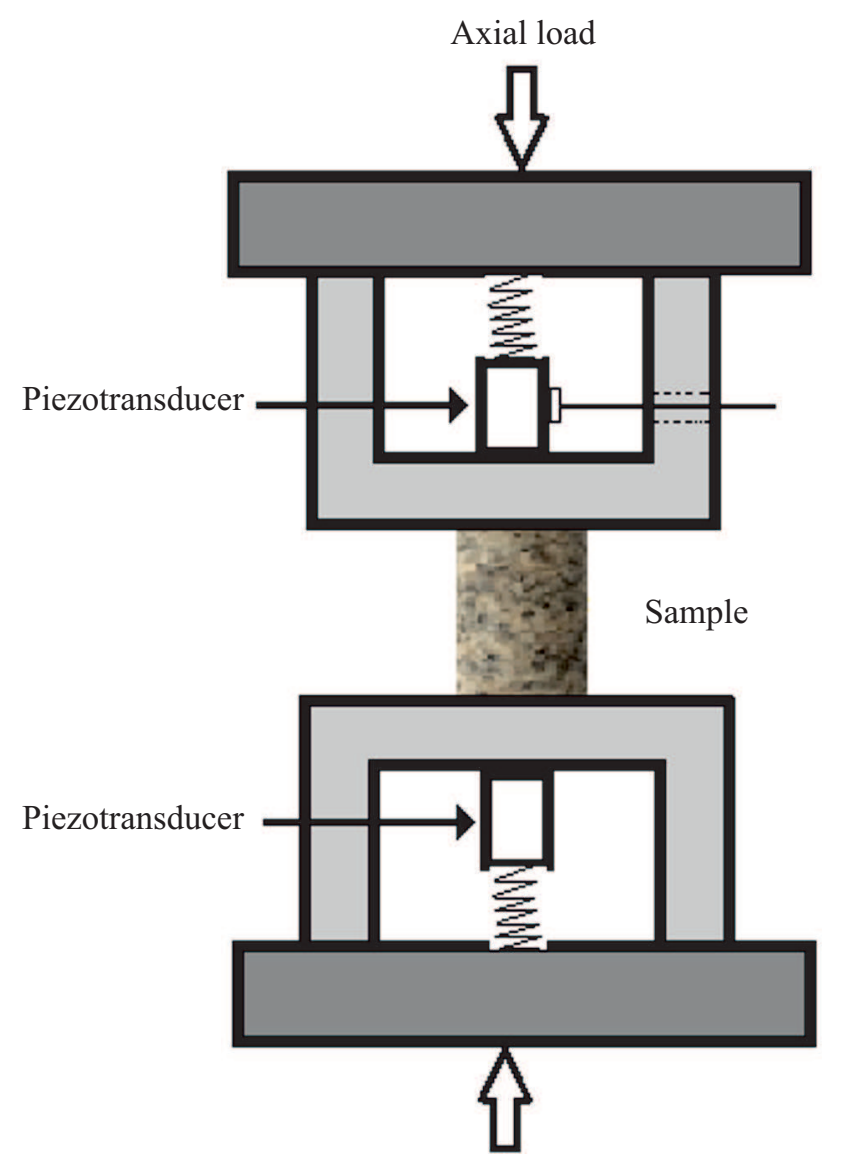

Рис. 1. Схема экспериментальной установки.

пьезопреобразователей позволило определять координаты источников событий АЕ (по высоте образца) с точностью не хуже $2 \mathrm{~mm}$. Перед началом эксперимента осуществлялась калибровка датчиков АЕ для проверки акустического контакта и определения характерной скорости звука.

Для исследования дефектной структуры образцов использовался томограф SkyScan 1172 (Bruker, Belgium), оснащенный микрофокусной рентгеновской трубкой Hamamatsu 100/250 с фокальным пятном $5 \mu$ m и $11 \mathrm{Mp}$ детектором. Сканирование образцов осуществлялось до и после механических испытаний. Продолжительность одной съемки составила $24 \mathrm{~h}$. Было проведено сканирование всей партии образцов песчаника до испытаний. Для дальнейших экспериментов отбирались те образцы, 


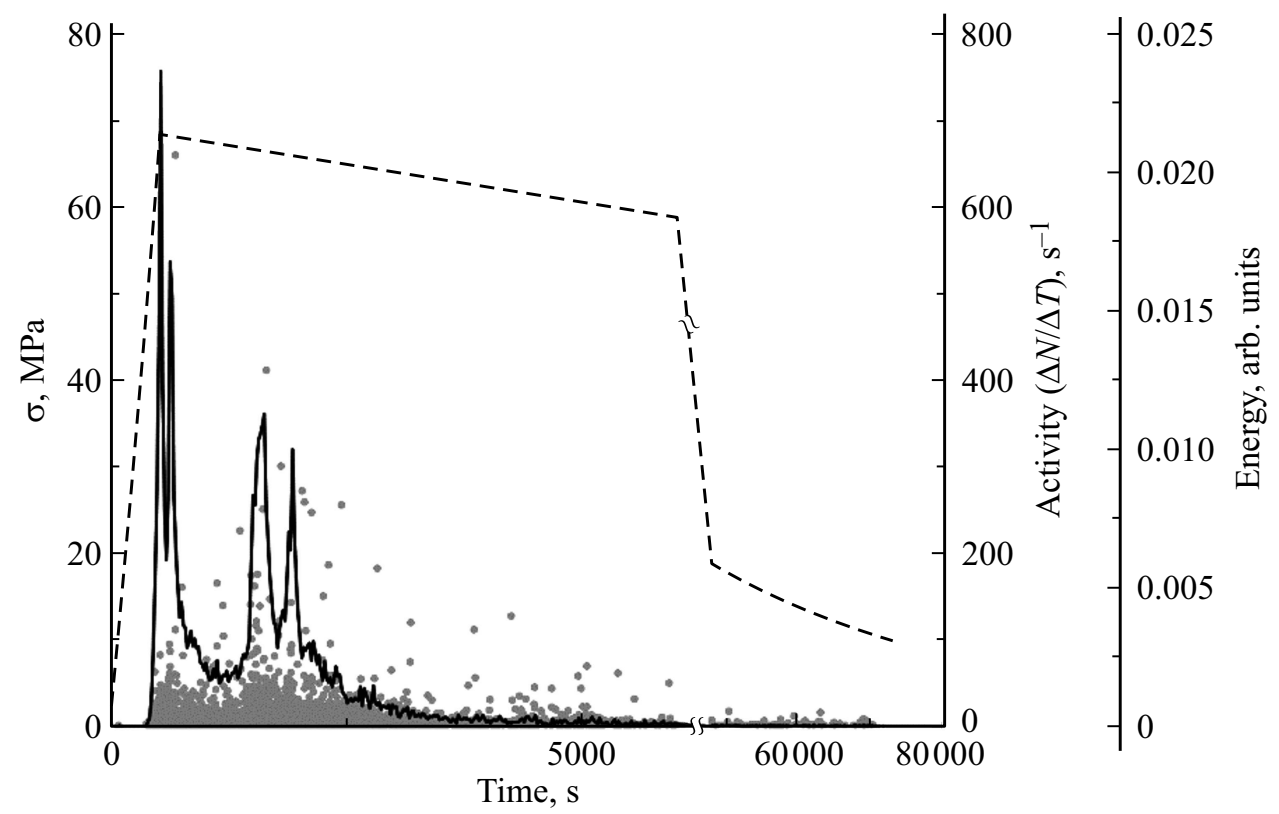

Рис. 2. Изменение напряжения (штриховая линия), активности акустической эмиссии (сплошная линяя) и энергии отдельных сигналов АЕ (точки) в процессе эксперимента.

в которых не было обнаружено структурных аномалий, которые могли стать концентраторами напряжений и источниками появления и развития разрушения. В результате были проведены испытания семи образцов песчаника Berea, в каждом эксперименте было зарегистрировано порядка 20000 сигналов АЕ, что достаточно для статистической обработки.

Выбранный размер образцов $(d=10 \mathrm{~mm}, h=20 \mathrm{~mm})$ позволил достигнуть пространственного разрешения компьютерной томографии $\sim 3 \mu \mathrm{m}$, что является максимально возможным для образцов данного размера с учетом физических принципов томографии, конструктивных особенностей рентгеновской трубки и камеры томографа [23].

\section{3. Результаты и обсуждение}

На рис. 2 представлены характерные временные зависимости приложенного напряжения, активности $\mathrm{AE}$ (число сигналов в единицу времени) и энергии сигналов акустической эмиссии. Видно, что активность акустической эмиссии значительно нарастает при подходе к максимальной приложенной нагрузке, а на втором этапе деформирования (удержания постоянной деформации) спадает со временем до нуля. При этом напряжение на образце экспоненциально спадало со временем.

Энергетическое распределение всех сигналов АЕ имеет степенной вид $\frac{\Delta N}{\Delta E}=a E^{-b}$ (с коэффициентом детерминации $R^{2}=0.95$ ) (рис. 3). Данный результат соответствует ранее установленным закономерностям $[24,25]$, согласно которым при приложенных напряжениях до $\approx 0.8-0.9 \sigma_{\text {destruction }}\left(\sigma_{\text {destruction }}-\right.$ разрушающее напря- жение) энергетическое распределение сигналов $\mathrm{AE}$ имеет экспоненциальный вид и соответствует неопасной стадии накопления дефектов. При напряжении $0.8-0.9 \sigma_{\text {destruction }}$ наблюдается смена функционального вида распределения с экспоненциального на степенной, что является индикатором начала критической „опасной“ стадии роста дефектов. Это позволяет говорить о том, что в данном эксперименте процесс дефектообразования соответствует опасной стадии.

Томографическая съемка образца песчаника, проведенная после завершения эксперимента, показала, что в результате нагружения в образце образовалась маги-

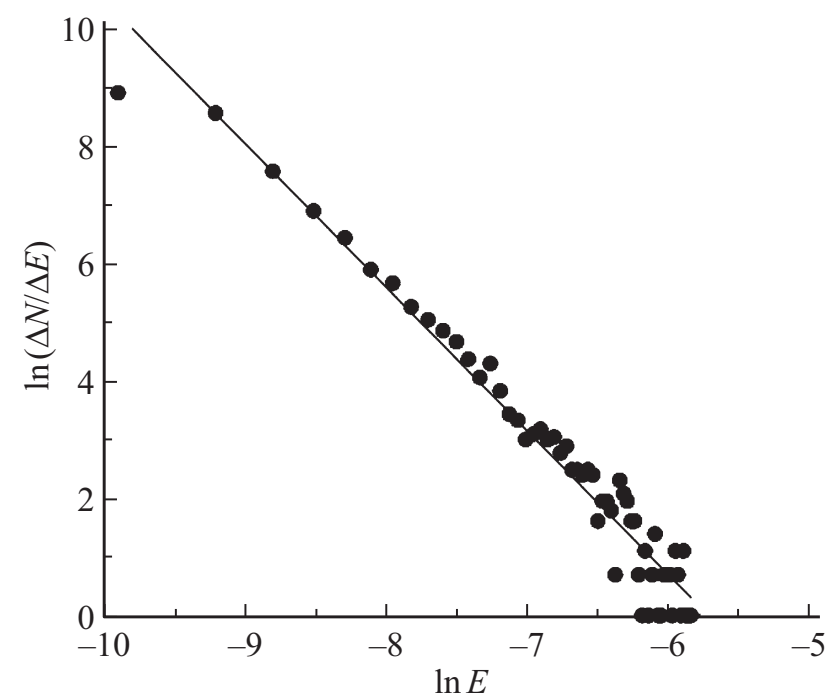

Рис. 3. Распределение по энергии всех сигналов АЕ, зарегистрированных в процессе нагружения. 


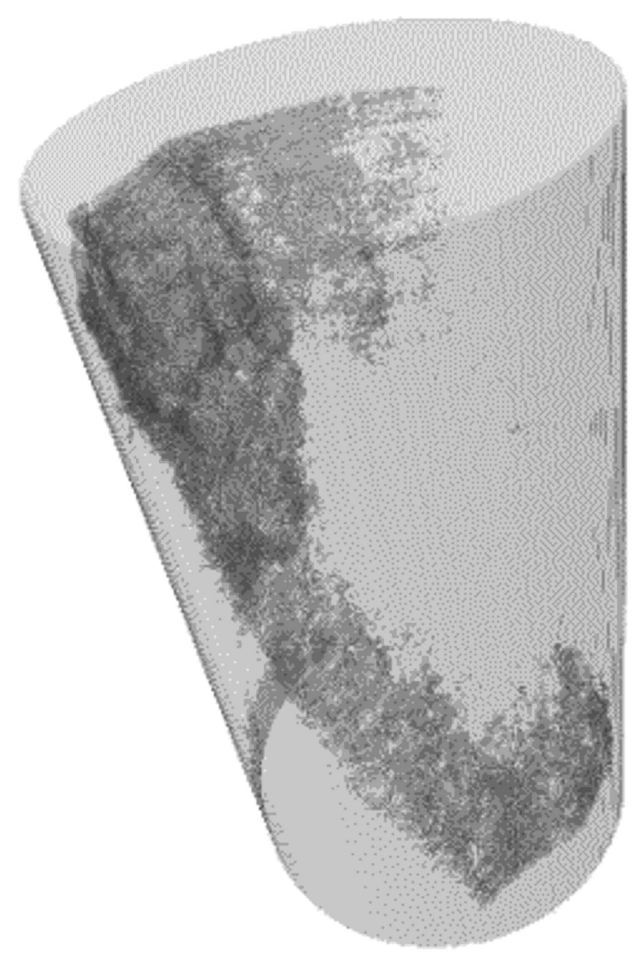

Рис. 4. Трехмерная визуализация дефектной структуры, построенная по данным рентгеновской томографии с использованием специализированного программного обеспечения СТan и CTvol.

стральная трещина, ориентированная относительно оси нагрузки под определенным углом (рис. 4). Формирование невертикальной трещины является типичным для случая одноосного сжатия хрупкого материала с невысоким уровнем бокового подпора (в нашем случае боковой подпор обеспечивался жесткой фиксацией упругой клейкой ленты на боковой поверхности образцов). Отметим, что вне области магистральной трещины, дефектов не обнаружено. Такой же результат был получен ранее $[26,27]$ при исследовании накопления дефектов в других природных гетерогенных материалах: граните Westerly и метапесчанике. Это позволяет говорить об общности результатов и их независимости от конкретного материала.

Для нивелирования влияния торцевых эффектов при дальнейшем анализе томографических и акустикоэмиссионных данных рассматривался только интервал высот от 4 до $14 \mathrm{~mm}$. В результате решения задачи линейной локации были определены координаты источников сигналов АЕ. На рис. 5 показана плотность распределения сигналов АЕ по координате (по высоте образца).

Видно, что наибольшее число сигналов АЕ зарегистрировано в области образца с координатами от 10 до $14 \mathrm{~mm}$. Это позволяет предположить, что в данной области происходило множественное образование дефектов. Использование двух методов неразрушающе- го контроля позволило нам сопоставить зависимости энергии сигналов $E_{\text {sum }}$ и объема дефектов от координаты (рис. 6). Объем дефектов вычислялся на основании данных рентгеновской компьютерной томографии с использованием специализированного программного пакета СТan в слоях высотой $2 \mathrm{~mm}$. Также для каждого из слоев была вычислена суммарная энергия сигналов AE, в нем расположенных. Из рис. 6 видно, что обе зависимости имеют подобный вид.

Полученные результаты позволили сопоставить между собой суммарную энергию $\mathrm{AE}\left(E_{\text {sum }}\right)$ и объем дефектов (рис. 7). Видно, что эта зависимость является линейной (с коэффициентом детерминации $\left.R^{2} \approx 0.98\right)$ и имеет вид

$$
E_{\text {sum }}=0.62 \mathrm{~V}-0.15 \text {. }
$$

Аналогичная зависимость для геологических масштабов была получена академиком Садовским [28] в середине

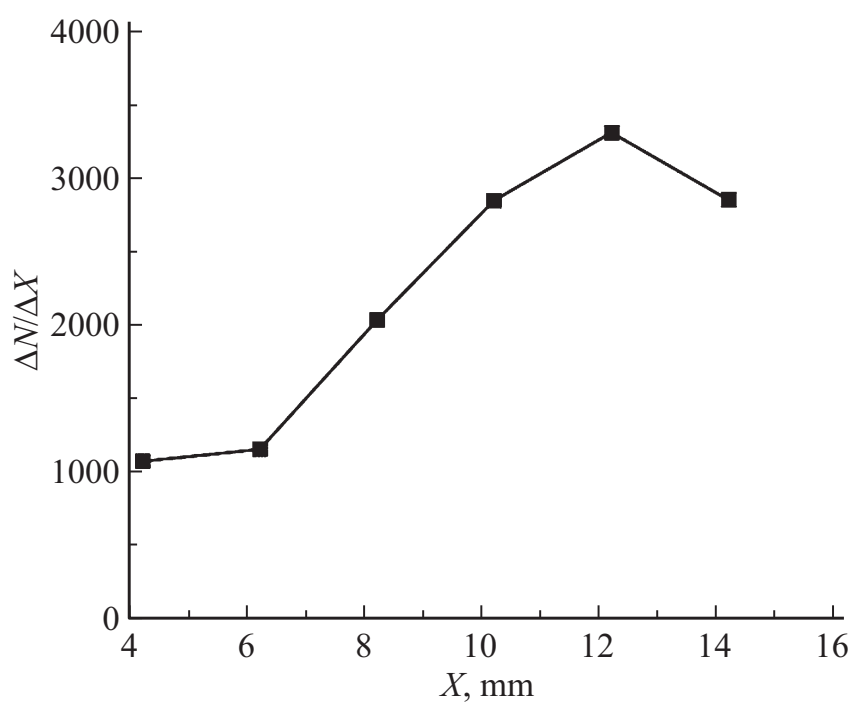

Рис. 5. Распределение числа сигналов АЕ по координате.

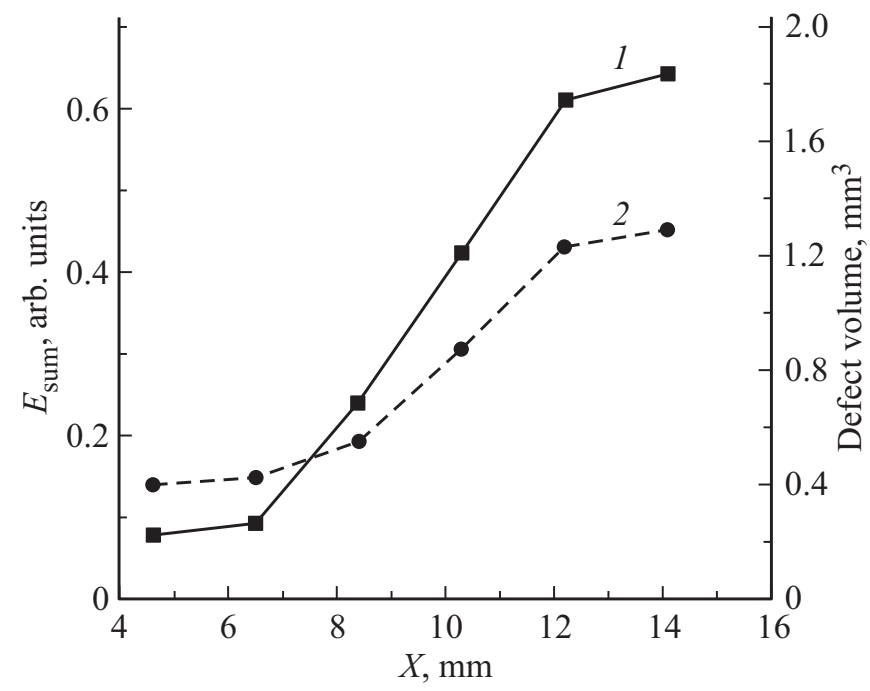

Рис. 6. Изменение суммарной энергии сигналов АЕ (кривая 1) и объема дефектов (кривая 2) по координате (высоте образца). 


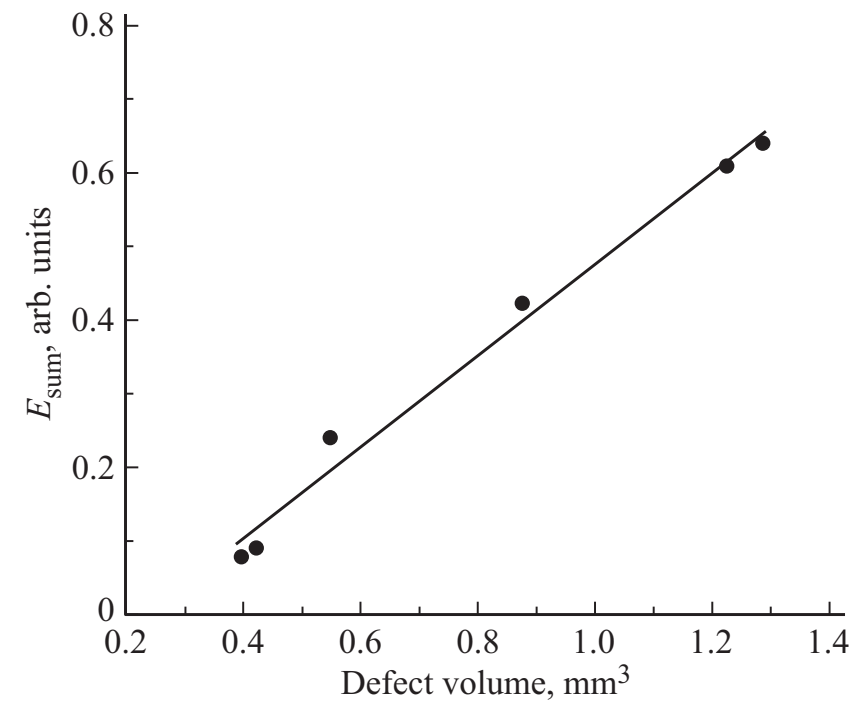

Рис. 7. Соотношение между суммарной энергией сигналов АЕ и объемом дефектов.

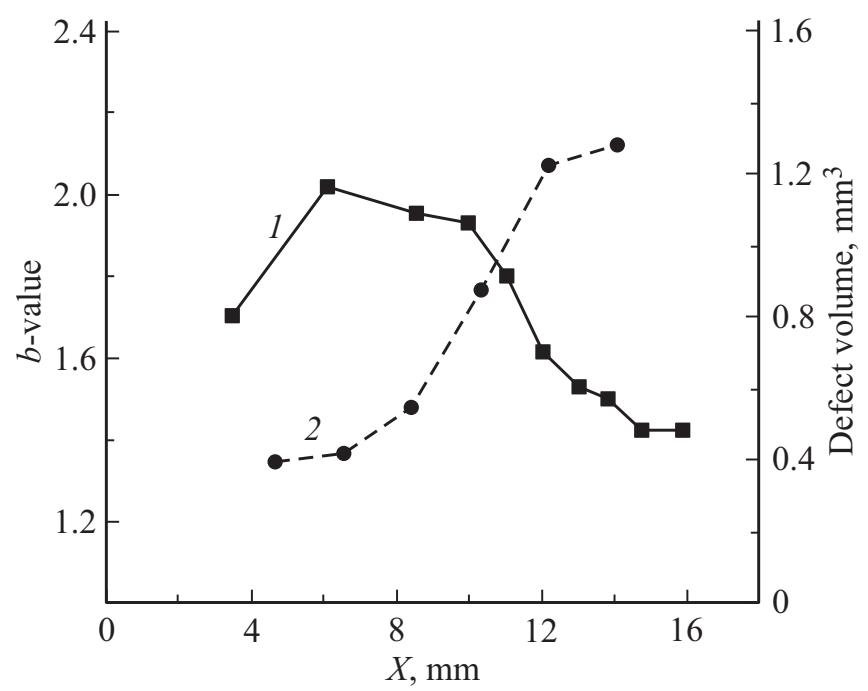

Рис. 8. Изменение коэффициента b-value (кривая 1) и объема дефектов (кривая 2) по координате (высоте образца).

прошлого века. Было показано, что энергия, выделяющаяся при землетрясении, прямо пропорциональна объему очага землятресения. Однако в лабораторных экспериментах получить подобную зависимость ранее не представлялось возможным.

Отметим, что объем дефектов в слое является интегральной характеристикой. Для оценки объема отдельного дефекта-источника сигнала $\mathrm{AE}$ необходимо провести нормировку на число сигналов. Тогда средний объем дефекта составит $\sim 10^{-4}-10^{-5} \mathrm{~mm}^{3}$.

Далее для изучения вариативности акустической эмиссии по координате (высоте образца) был проведен анализ изменения показателя степени энергетического распределения сигналов AE - так называемый b-value анализ [29-32], результаты которого представлены на рис. 8. Энергетические распределения строились для последовательных по координате выборок, состоящих из фиксированного числа событий. Из рисунка видно, что показатель b-value и объем дефектов изменяются в противофазе. Такое поведение согласуется с установленной нами линейной зависимостью суммарной энергии сигналов АЕ от объема дефектов. Рост коэффициента b-value в области от 4 до $5 \mathrm{~mm}$ указывает на преобладание низкоэнергетических событий, источниками которых, по-видимому, являются малоразмерные дефекты. Постепенное уменьшение значения b-value соответствует образованию более крупных дефектов, что коррелирует с увеличением объемной доли дефектов в этой пространственной области (от 6 до $16 \mathrm{~mm}$ ).

Результаты компьютерной томографии и данные акустической эмиссии могут быть использованы для оценки характерного линейного размера одиночных дефектов, образующихся при деформировании образца. Такая оценка требует априорной гипотезы о форме образующихся дефектов. Следуя работам [33-36], предположим, что образующиеся дефекты имеют форму:

- шара с диаметром $D_{1}=\sqrt[3]{\frac{6 V}{\pi}}$;

- вытянутого эллипсоида с наибольшей полуосью $D_{2}=\frac{3 V}{4 \pi b^{2}}$, где $b-$ размер средней и малой полуосей;

- круговой трещины диаметром $D_{3}=\sqrt{\frac{4 V}{\pi h}}$, где $h-$ толщина трещины.

Для вычисления характерного линейного размера в случае вытянутого эллипсоида и круговой трещины предположим, что размеры $b$ и $h$ в 2 раза меньше наибольшей полуоси и толщины. Оценим характерный линейный размер на основе суммарного объема всех образовавшихся дефектов $V_{\text {sum }}$, нормированного на количество сигналов АЕ, и суммарной энергии этих сигналов. В результате, для дефектов различной формы получаем следующие характерные размеры:

$$
\begin{aligned}
& D_{1} \approx 88 \mu \mathrm{m}, \\
& D_{2} \approx 69 \mu \mathrm{m}, \\
& D_{2} \approx 69 \mu \mathrm{m},
\end{aligned}
$$

которые отличаются друг от друга не более чем на $30 \%$. Таким образом, вне зависимости от принятой гипотезы о характерной форме источника АЕ его средний линейный размер не превышает $100 \mu \mathrm{m}$. Полученный результат коррелирует с оценкой, построенной на основе известных скейлинговых соотношений для магнитуды, выделившейся энергии и объема очага землетрясения. Так, средняя магнитуда сигнала АЕ равна -9 [18] и связана с энергией, выделившейся в источнике, соотношением [37]:

$$
\lg E=1.8 M+4
$$


В свою очередь объем источника может быть оценен по величине выделившейся энергии (согласно [28]) как

$$
\lg V=\lg E-3
$$

что приводит к оценке объема одиночного дефекта $V \sim 10^{-4}-10^{-5} \mathrm{~mm}^{3}$. Данная оценка полностью соответствует величинам, полученным по данным рентгеновской компьютерной томографии в настоящей работе. Необходимо заметить, что преимущество оценки, полученной в настоящее работе, заключается в большей точности, а главное, в возможности по найденному соотношению (1) для конкретного материала оценивать характерный линейный размер дефектов непосредственно в процессе контроля (при эксплуатационных или аварийных нагрузках).

\section{4. Заключение}

С использованием методов акустической эмиссии и рентгеновской компьютерной томографии проведено экспериментальное исследование закономерности накопления повреждений при одноосном сжатии образцов песчаника Berea. В результате анализа внутренней структуры песчаника после деформирования, полученной с помощью рентгеновской компьютерной томографии, показано, что все дефекты локализованы в области магистральной трещины. На основе данных томографии построено распределение объема микродефектов по высоте образца, удовлетворительно коррелирующее с распределением источников АЕ по высоте. Проведено сопоставление пространственных распределений объема микродефектов и суммарной энергии сигналов $\mathrm{AE}$, в результате которого показана линейная зависимость между этими параметрами.

На основе априорных гипотез о форме образующихся дефектов оценен характерный линейный размер дефектов - источников АЕ, не превышающий $100 \mu \mathrm{m}$. Полученный результат имеет существенное прикладное значение с точки зрения in situ оценки характерных размеров (объема) дефектов по данным акустической эмиссии, образующихся в процессе эксплуатации объекта контроля.

\section{Финансирование работы}

Работа выполнена при финансовой поддержке Российского фонда фундаментальных исследований (грант 1905-00248).

\section{Конфликт интересов}

Авторы заявляют, что у них нет конфликта интересов.

\section{Список литературы}

[1] T.H.W. Goebel, T.W. Becker, D. Schorlemmer, S. Stanchits, C. Sammis, E. Rybacki, G. Dresen. J. Geophys. Res. 117, B03310 (2012).

[2] В.И. Веттегрень, В.С. Куксенко, И.П. Щербаков. ЖТФ 83, 1, 144 (2013).

[3] A.S. Voznesenskii, M.N. Krasilov, Ya.O. Kutkin, M.N. Tavostin, Yu.V. Osipov. Int. J. Fatigue 97, 70 (2017).

[4] Acoustic, electromagnetic, neutron emissions from fracture and earthquake / Ed. A. Carpinteri, G. Lacidogna, A. Manuello. Springer (2015). 265 p.

[5] A.G. Vostretsov, G.I. Kulakov, A.A. Bizyaev, G.E. Yakovitskaya. J. Mining Sci. 53, 6, 1152 (2017).

[6] V.N. Oparin, G.E. Yakovitskaya, A.G. Vostretsov, V.M. Seryakov, A.V. Krivetsky. J. Mining Sci. 49, 3, 343 (2013).

[7] L. Wu, J. Wang. J. Mining Sci. 35, 969 (1998).

[8] L. Ma, H. Sun. Infrared Phys. \& Technology 3, 144 (2018).

[9] L. Phono. Eng. Fracture Mech. 35, 291 (1990).

[10] H. Sun, X.L. Liu, S.G. Zhang, K. Nawnit. Eng. Fracture Mech. 226, 106845 (2020).

[11] Kang Zhao, Xiang Yu, Yun Zhou, Qing Wang, Junqiang Wang, Jiaolong Hao. Int. J. Rock Mech. Mining Sci. 132, 104392 (2020).

[12] F. Gao, H. Kang, L. Yang. Sci. Rep. 10, 13422 (2020).

[13] Song Luo, Fengqiang Gong. Eng. Fracture Mech. 234, 107102 (2020).

[14] Kang Peng, Shaowei Shi, Quanle Zou, Junhui Mou, Jin Yu, Yongjiang Zhang, Yanying Cheng. Energy Sci. Eng. https://doi.org/10.1002/ese3.738 (2020).

[15] Santosh G. Shah, J.M. Chandra Kishen. Eng. Fracture Mech. 87, 36 (2012).

[16] S. Yuyama, Z.-W. Li, M. Yoshizawa, T. Tomokiyo, T. Uomoto. NDT \& International 34, 381 (2001).

[17] Youngguk Seo, Y. Richard Kim. KSCE J. Civil Eng. 12(4), 237 (2008).

[18] Xinglin Lei, Shengli Ma. Earthquake Sci. 27, 627 (2014).

[19] Junwen Zhang. Hindawi. Shock and Vibration. 2018, Article ID 3057628, (2018).

[20] Л.Р. Ботвина. Физика Земли 10, 5 (2011).

[21] Л.Р. Ботвина Разрушение: кинетика, механизмы, общие закономерности. Наука, М. (2008). 334 с.

[22] P. Churcher, P. French, J. Shaw. SPE International Symposium on Oileld Chemistry. SPE21044 (1991).

[23] Teodor Toth, Radovan Hudak. Acta Mech. Slovaca 17 (4), 40 (2013).

[24] E. Damaskinskaya, D. Frolov, D. Gafurova, D. Korost, I. Panteleev. Interpretation 5, 4, SP1 (2017).

[25] Е.Е. Дамаскинская, И.А. Пантелеев, Д.Р. Гафурова, Д.И. Фролов. ФТТ 60, 7, 1353 (2018).

[26] Е.Е. Дамаскинская, В.Л. Гиляров, И.А. Пантелеев, Д.Р. Гафурова, Д.И. Фролов. ФТТ 60, 9, 1775 (2018).

[27] E. Damaskinskaya, V. Hilarov, I. Panteleev, D. Korost, D. Frolov. In: Proceedings in Earth and Environmental Sciences. The 5th Int. Conf. „Trigger Effects in Geosystems“. Moscow, Russia (4-7 June, 2019). Springer (2019). P. 23.

[28] М.А. Садовский. Докл. АН СССР 275, 5, 1087 (1984).

[29] Л.Р. Ботвина, А.П. Солдатенков, М.Р. Тютин. Докл. РАН 446, 3, 325 (2012). 
[30] M.V.M.S. Rao, K.J. Prasanna Lakshmi. Current Sci. 89, 9, 1577 (2005).

[31] S. Colombo, I.G. Main, M.C. Forde. J. Mater. Civil Eng. 15, 3, 280 (2003).

[32] A. Carpinteri, G. Lacidogna, S. Puzzi. Chaos. Solitons Fractals 41, 2, 843 (2009).

[33] S. Hirose, J. Achenbach. Eng. Fracture Mech. 39, 1, 21 (1991).

[34] O.Y. Andreykiv, M.V. Lysak, O.M. Serhiyenko, V.R. Skalsky. Eng. Fracture Mech. 68, 11, 1317 (2001).

[35] M. Lysak. Eng. Fracture Mech. 55, 3, 443 (1996).

[36] A. Bizzarri. Rev. Geophys. 49, RG3002 (2007). doi: 10.1029/2011RG000356

[37] T.G. Rautian, V.I. Khalturin, K. Fujita, K.G. Mackey, M.L. Nichols. Seismological Res. Lett. 78, 6, 579 (2007).

Редактор К.В. Емиев 